Method We carried out the research by multi-steps random sampling through questionaires. 1312 records had been completed for analysis. Indicators as smoking, age, education, occupation and income, etc., were calculated.

Results The total smoking rate was $23.8 \%, 52.4 \%$ in male and $4.0 \%$ in female. The smoking rates of different education, occupation and income had statistical differences.

Conclusion The smoking rate of Shougang district was lower than that of Beijing and the whole country. The smoking rate in male was significantly higher than that in female. It is very important to develop the health education and the smoking control in some special people.

\section{Q0263 EPIDEMIOLOGICAL SURVEY OF HYPERTENSION IN GENERAL ADULT POPULATION OF DIFFERENT NATIONALITIES IN XINJIANG}

doi:10.1136/hrt.2010.208967.263

Huang Ying, Ma Yitong, Liu Fen, Yang Yining, Li Xiao-Mei, Sun Minghui, Yao Juan, An Yong, Yao Juan An Yong

Objective To estimate the prevalence and distribution of hypertension and to determine the status of hypertension awareness, treatment, and control in Xinjiang.

Methods The Cardiovascular Risk Survey in Xinjiang, A total of 14618 adults, age $\geq 35$ years in Xinjiang.

Results The standardisation prevalence is $42.25 \%$. Prevalence rate of hypertension is $42.44 \%$ in Han, $35.2 \%$ in Uygur, $53.04 \%$ in Kazak respectively. Among hypertensive patients, only $45.5 \%$ were aware of their high blood pressure, $19.7 \%$ were taking antihypertensive medication, and $11.3 \%$ achieved blood pressure control $(<140 /$ $90 \mathrm{~mm} \mathrm{Hg}$ ). These data are $46.7 \%, 29.6 \%, 6.1 \% ; 48.5 \%, 18.3 \%$, $14.2 \% ; 41.5 \%, 14.5 \%, 4.6 \%$ as sequence in Han, Uygur and Kazak. Conclusions Our results indicate that hypertension is highly prevalent in Xinjiang. The percentages of those with hypertension who are aware, treated, and controlled are relatively low.

\section{e0264 INVESTIGATION ON BLOOD LIPIDS OF THE LI NATIONALITY IN HAINAN ISLAND}

doi:10.1136/hrt.2010.208967.264

Yunbo Zhang, Zhen Yao, Yong Zhang, Ying Li, Ruilian Ma, Shijiao Yan. The People's Hospital of Sanya

Objective To study the blood lipid level of the Li nationality in Hainan Island, and to compare it between Li nationality and other people. To analyse the effects of the way of life style and the Polymorphisms of blood lipid gene on the levels of blood lipids.

Methods The epidemiological study was carried out in a natural population 1000 (500 samples from the Li nationality and 500 samples from the Han nationality) individuals aged aged between 30 and 50 from Li Nationality in Hainan area by cluster and random sampling. To measure TC, TG, LDL-C, HDL-C according to "Suggestion on protocol of measuring serum lipdaemia and standardisation", the data were analysed by SPSS 13.0.

Results There were no differences about the levels of TC, TG, HDL$\mathrm{C}$ and LDL-C between the Li nationality and the Han nationality, but there were significant difference about the levels of HDL-C between two population $(p<0.05)$. The levels of HDL-C in the Li nationality was significantly higher than that in the Han nationality among female $(p<0.05)$, and the levels of TG in the Han nationality was significantly higher than that in the Li nationality among male $(p<0.05)$. The levels of HDL-C in the Li nationality was significantly higher than that in the Han nationality in the group of age 30-39 and the group of age $40-49(p<0.05)$.
Conclusions There were statistical difference about the levels of the blood lipids between the $\mathrm{Li}$ and the Han nationality. The reason could be that the Li nationality living in the poor area who are under low living standard. The different Polymorphisms of blood lipid genes may be one of the causes.

\section{e0265 ANALYSIS OF 24 H SLEEP DEPRIVATION ON ARRHYTHMIA AND HEART RATE VARIABILITY}

doi:10.1136/hrt.2010.208967.265

Wei-Ren Chen, Xiang-Min Shi. Pla General Hospital

Backgroud Sleep deprivation (SD) is a common phenomenon in emergency, especially during war time, conflict, natural disasters or disease crisis such as SARS, it can cause exhaustion in members of armed forces and non-combat casualty. In conditions of high-tech war, deprivation is becoming more serious and mandatory than ever before.

Objective To study the impact of 24-h sleep deprivation on arrhythmia and heart rate variability (HRV).

Methods Soldiers were used as research objects, they were divided into normal group and the sleep deprivation group. 1. Heart rate, atrial premature beat, ventricular premature beat and heart rate variability were observed by $24-\mathrm{h}$ ambulatory ECG during sleep deprivation. 2. The changes of serum cortisol levels were detected during sleep deprivation.

Results 1. After $24 \mathrm{~h}$ sleep deprivation, HF, SDNN and RMSSD significantly reduced, LF significantly increased, LF /HF showed a significant increase. 2. Part of volunteers presented atrial premature beats and ventricular premature beats. 3 . The level of serum cortisol significantly increased.

Conclusion The underlying mechanism of alteration of arrhythmia and HRV after 24-h sleep deprivation would be ascribed to lower vagal activity and elevated sympathetic activity.

\section{E0266 SNORING IS ASSOCIATED WITH SUBCLINICAL CAROTID ATHEROSCLEROSIS IN 1050 URBAN CHINESE}

doi:10.1136/hrt.2010.208967.266

Li Yan, Zhao Dong, Liu Jing, Wang Wei. Capital Medical University Affiliated Beijing Anzhen Hospital, Beijing Institute of Heart, Lung and Blood Vessel Diseases

Context Epidemiological studies have identified snoring as a risk factor for atherosclerotic cardiovascular diseases. However, there is little evidence on snoring and subclinical atherosclerosis.

Objective To evaluate whether and to what extent snoring is associated with carotid atherosclerosis.

Methods Population-based study was conducted at a community in Beijing on 1050 subjects aged 50-79 years who had an ultrasound examination of the carotid artery at age ranging from 45 to 74 years in 2002 and a carotid ultrasonic reexamination in 2007, as well as a cross-sectional survey including snoring information and cardiovascular risk factors from September to November 2007.

Main Outcome Measures Carotid intima-media thickness (IMT) and plaque as two indexes of carotid atherosclerosis were diagnosed by B-mode ultrasonography. Association of snoring with increased IMT and plaque were analysed by multivariable logistic regression models adjusted for cardiovascular risk factors.

Results The prevalence of snoring was $64.3 \%$ in this population (71.4\% in males and $58.4 \%$ in females). In multivariable models adjusted for traditional risk factors, snoring was significantly associated with increased IMT of common carotid artery (CCA) (OR, 1.38; 95\% CI 1.04 to 1.82 ) and bifurcated carotid artery (BCA) (OR, 1.65 ; $95 \%$ CI 1.24 to 2.19 ), with having plaque of CCA (OR, 